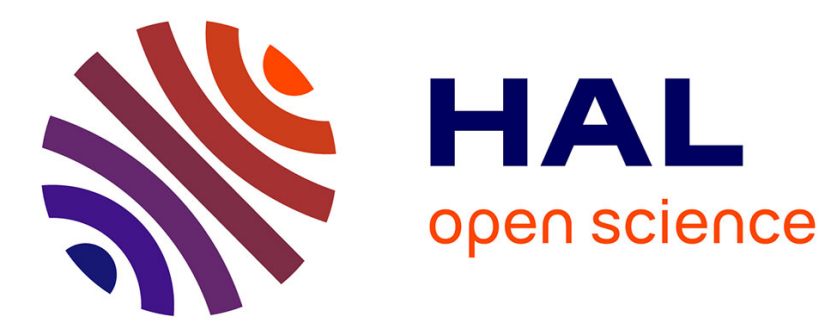

\title{
Chaos in eigenvalue search methods
}

D. Mancusi, Andrea Zoia

\section{- To cite this version:}

D. Mancusi, Andrea Zoia. Chaos in eigenvalue search methods. Annals of Nuclear Energy, 2017, 112, pp.354-363. 10.1016/j.anucene.2017.10.022 . cea-02421744

\section{HAL Id: cea-02421744 https://hal-cea.archives-ouvertes.fr/cea-02421744}

Submitted on 6 Mar 2020

HAL is a multi-disciplinary open access archive for the deposit and dissemination of scientific research documents, whether they are published or not. The documents may come from teaching and research institutions in France or abroad, or from public or private research centers.
L'archive ouverte pluridisciplinaire HAL, est destinée au dépôt et à la diffusion de documents scientifiques de niveau recherche, publiés ou non, émanant des établissements d'enseignement et de recherche français ou étrangers, des laboratoires publics ou privés. 


\title{
Chaos in eigenvalue search methods
}

\author{
Davide Mancusi ${ }^{\mathrm{a}}$, Andrea Zoia ${ }^{\mathrm{a}, *}$ \\ ${ }^{a}$ Den-Service d'études des réacteurs et de mathématiques appliquées (SERMA), CEA, \\ Université Paris-Saclay, F-91191, Gif-sur-Yvette, France
}

\begin{abstract}
Eigenvalue searches for multiplying systems emerge in several applications, encompassing the determination of the so-called alpha eigenvalues associated to the asymptotic reactor period and the adjustment of albedo boundary conditions or buckling in assembly calculations. Such problems are usually formulated by introducing a free parameter into a standard power iteration, and finding the value of the parameter that makes the system exactly critical. The corresponding parameter is supposed to converge to the sought eigenvalue. In this paper we show that the search for the critical value of the parameter might fail to converge for deep sub-critical systems: in this case, the search algorithm may undergo a series of period doubling bifurcations (leading to a multiplicity of solutions) instead of converging to a fixed point, or it may even crash. This anomalous behaviour is explained in terms of the mathematical structure of the search algorithm, which is shown to be closely related to the well-known logistic map for a few relevant applications illustrated in the context of the rod model. The impact of these findings for real-life applications is discussed, and possible remedies are finally suggested.
\end{abstract}

Keywords: Eigenvalues, Chaos, Power iteration, Monte Carlo, TrIPOLI- $4{ }^{\circledR}$

\section{Introduction}

A large class of problems emerging in nuclear reactor physics involve searching for the stationary state of a multiplying system by adjusting a control parameter (Bell and Glasstone, 1970, Azmy and Sartori, 2010). Examples include for instance the determination of the value of boron dilution or the control rod

${ }^{*}$ Corresponding author. Tel. +33 (0)1 69087976

Email address: andrea.zoia@cea.fr (Andrea Zoia) 
position that make the reactor exactly critical, all the other system parameters being kept constant. Similar searches are quite common also outside the realm of reactor control. Consider, for instance, the determination of the equilibrium temperature profile of reactor cores with thermal-hydraulics feedback. The sta10 tionary solution of the coupled problem may be sought by alternatively iterating neutron transport and thermal-hydraulics solvers until they converge to a fixed point. There are no external control parameters in this case, but the temperature field may be seen to play the role of an adjustable parameter, whose shape at convergence makes the reactor critical. Other examples of critical parameter

15 searches with iterative solution schemes arise in the context of the determination of the albedo at the core boundaries (Cho et al., 2009; Yun and Cho, 2009) or in reactor period calculations (Azmy and Sartori, 2010; Zoia et al., 2015; Nauchi, 2014).

Although inspired by their real-life counterparts, for the purpose of this paper we will regard critical parameter searches as mathematical problems: we will assume that the reactor state can be described by the $k$-eigenvalue form of the linear Boltzmann equation (Goad and Johnston, 1959; Lewis and Miller, 1984; Lux and Koblinger, 1991), with a single free control parameter $p$. The presence of multiple control parameters, and the possible interactions between each other due to coupling mechanisms, will be neglected. For any value of $p$, there will be a spectrum $\sigma_{p}[k]$ of eigenvalues associated to the Boltzmann equation: starting from an arbitrary initial condition, the reactor will ultimately relax to its fundamental mode $\varphi_{k_{0}(p)}$, with associated fundamental eigenvalue $k_{0}(p)$. Both $\varphi_{k_{0}(p)}$ and $k_{0}(p)$ depend on the control parameter $p$. Formally speaking, the critical solution is usually sought by introducing iterative update schemes for the control parameter: the $k$-eigenvalue equation is solved for the fundamental eigenvalue $k_{0}(p)$ for a given $p$ (for instance by power iteration), then $p$ is progressively adjusted based on the value $k_{0}(p)$. If such a scheme ultimately converges to a fixed point, with corresponding fundamental eigenvalue $k_{0}(p)=k_{0}\left(p_{c}\right)=1$, the resulting value of the control parameter is the sought solution $p=p_{c}$ that makes the system exactly critical.

One may be tempted to assume that such iterative schemes always converge to the fixed point $\left\{p=p_{c}, k_{0}\left(p_{c}\right)=1\right\}$, assuming of course that it exists. Clearly, though, if the initial conditions of the iterative scheme are poorly chosen, the search may diverge or enter non-physical regions of the search space. Under these conditions, the fixed point $p_{c}$ is never attained. However, even if a solution exists and suitable initial conditions are selected, the iterative scheme may still fail to converge. This phenomenon has been repeatedly observed and is 
widely documented in the literature related to the numerical determination of the asymptotic reactor period (the so-called $\alpha-k$ iteration). For instance, simple iterative schemes for $\alpha$ eigenvalue search lead to abnormal code termination in sub-critical configurations (Hill, 1983). The same issue was later reported by many authors, and effective solutions have been proposed by resorting to operator or eigenvalue shifting techniques (Yamamoto and Miyoshi, 2003; Ye et al. 2006; Zoia et al., 2014, 2015).

It is perhaps worth observing that critical parameter searches are essentially root-finding problems for numerical functions. Thus, the whole apparatus of numerical root-finding methods can be in principle brought to bear in order to determine the value of the parameter $p$ that makes the reactor critical. Many root-finding algorithms do guarantee convergence under weak assumptions. For instance, the class of bracketing methods (such as the bisection method or the regula falsi method) guarantee convergence if the initial conditions are suitably chosen (Traub, 1964; Householder, 1970; Ortega and Rheinboldt, 1970). However, it is not straightforward to apply bracketing methods to stochastic rootso finding problems (Pasupathy and Kim, 2011).

The purpose of this paper is to provide insight into the reasons why some iterative schemes for criticality searches may fail to converge to the fixed-point solution, even when the latter exists and even when the search is seeded with appropriate initial conditions. While this paper addresses these questions in the $k_{0}(p)$ and for the adjustment of the control parameter $p$, we actually also have in mind possible applications to eigenvalue searches with Monte Carlo codes. In this case, $k_{0}(p)$ is determined by the stochastic implementation of power iteration (by running a large number of cycles corresponding to successive neutron generations) and $p$ is updated at the end of each cycle. In view of this consideration, we will mostly focus on update rules for $p$ simple enough to depend on the value of $k_{0}(p)$ at the current cycle, without the need of storing in memory the past cycles. For the same reason, we will not consider methods based on derivatives of $k_{0}(p)$, which cannot be straightforwardly estimated in Monte Carlo schemes.

This paper is organized as follows. In Sec. 2 we begin by introducing the required notation and providing the general mathematical setup for eigenvalue searches. In particular, we will show that these problems can be formally recast into a discrete dynamical system, whose equilibrium point is the sought solution. In Sec. 3 we will provide a few numerical illustrations of eigenvalue searches in the context of the rod model, a simple system involving mono-energetic neutron transport. A broad class of eigenvalue problems associated to the rod model 
will be examined. We will show in particular under which conditions these searches may fail to converge, and display instead an oscillatory or even chaotic behaviour. Then, in Sec. 4 we will show that the mechanisms that lead to the

85 failure of the eigenvalue search have a universal character (whose origins will be elucidated) and might thus more broadly emerge in real-life applications. Some remedies especially conceived in order to regularize the eigenvalue searches and possibly suppress the route to instabilities and chaos will be proposed and numerically tested in Sec. 5. Conclusions will be finally drawn in Sec. 6 .

\section{Definitions and notation}

To fix the ideas, let us assume that the state of the reactor can be characterized in terms of the $k$-eigenvalue form of the linear Boltzmann equation, namely,

$$
\left\{\begin{array}{l}
\mathcal{L} \varphi_{k}=\frac{1}{k} \mathcal{F} \varphi_{k}, \\
\text { B. C. on } \varphi_{k}
\end{array}\right.
$$

where $\varphi_{k}=\varphi_{k}(\mathbf{x}, \boldsymbol{\Omega}, E)$ are the eigenfunctions of the angular neutron flux and $k$ the associated eigenvalues,

$$
\mathcal{L}=\boldsymbol{\Omega} \cdot \nabla_{\mathbf{x}}+\Sigma_{t}(\mathbf{x}, E)-\int d \mathbf{\Omega}^{\prime} \int d E^{\prime} \Sigma_{s}\left(\mathbf{x}, E^{\prime}\right) f_{s}\left(\mathbf{\Omega}^{\prime}, E^{\prime} \rightarrow \mathbf{\Omega}, E\right)
$$

95 is the net disappearance operator, with $\Sigma_{t}$ the total cross section, $\Sigma_{s}$ the scattering cross section, and $f_{s}$ the scattering kernel, and

$$
\mathcal{F}=\frac{\chi_{f}(E)}{4 \pi} \int d \mathbf{\Omega}^{\prime} \int d E^{\prime} v_{f}\left(E^{\prime}\right) \Sigma_{f}\left(\mathbf{x}, E^{\prime}\right)
$$

is the fission operator, with $\Sigma_{f}$ the fission cross section, $v_{f}$ the average number of fission neutrons, and $\chi_{f}$ the fission spectrum (Bell and Glasstone, 1970). Boundary conditions (B. C.) on $\varphi_{k}$ must be also assigned for Eq. (1). Although the existence of a dominant discrete eigenvalue $k_{0}$ with real part larger than those of all other eigenvalues in the spectrum $\sigma[k]$ and with non-negative associate eigenfunction $\varphi_{k_{0}}$ has not been proven for arbitrary operators $\mathcal{L}$ and $\mathcal{F}$, domain shapes and boundary conditions, under rather mild assumptions it is reasonable to assume that the fundamental eigenpair $\left\{\varphi_{k_{0}}, k_{0}\right\}$ exists (Lewis and Miller, 1984; Lux and Koblinger, 1991). This means that the neutron population in the core, starting from arbitrary initial conditions, will eventually relax to a phase space 
distribution proportional to $\varphi_{k_{0}}$, and $k_{0}$ will asymptotically yield the ratio between population sizes at two successive generations. If $k_{0}>1$ the population will diverge; if $k_{0}<1$ the population will shrink; and if $k_{0}=1$ the population will stay constant, which precisely defines the critical state.

In many practical applications, one is only interested in determining the asymptotic behaviour of the core, the precise shape of the spectrum $\sigma[k]$ being of lesser importance. In this case, a widely adopted technique for assessing the dominant eigenpair $\left\{\varphi_{k_{0}}, k_{0}\right\}$ of Eq. (1) is the power iteration (Lewis and Miller, 1984; Lux and Koblinger, 1991). This method requires an ansatz $\varphi_{k}^{(0)}$ for the angular flux, and eventually converges to $\left\{\varphi_{k_{0}}, k_{0}\right\}$ by iterated application of the update rule

$$
\varphi_{k}^{(n+1)}=\frac{1}{k^{(n)}} \mathcal{L}^{-1} \mathcal{F} \varphi_{k}^{(n)},
$$

with $k^{(n)}=\left|\varphi_{k}^{(n)}\right| /\left|\varphi_{k}^{(n-1)}\right|$ and $k^{(0)}=1$, and the same boundary conditions as in Eq. (11). For sufficiently large $n$, the eigenvalue $k^{(n)}$ converges to $k_{0}$ as $k^{(n)} \simeq$ $k_{0}+c\left(\frac{k_{1}}{k_{0}}\right)^{n}+\cdots$, where $c$ is a problem-dependent constant and $k_{1}$ is the eigenvalue corresponding to the first excited eigenmode. Correspondingly, the function $\varphi_{k}^{(n)}$ converges to the fundamental eigenmode $\varphi_{k_{0}}$. The power iteration scheme given in Eq. (4) can be solved by either deterministic or Monte Carlo methods (Lewis and Miller, 1984; Lux and Koblinger, 1991).

125 In the following, we will exclusively focus on determining the fundamental eigenvalue, so that we will drop the subscript 0 from both the eigenvalue and the associated eigenfunction.

Generally speaking, the operators in Eq. (1) and the boundary conditions may both depend on a control parameter $p$, representing the effects of an external control element (such as a control rod or a reflector layer), or a physical counter-reaction (such as temperature or material density variations) in response to deviations from the nominal reactor state. This yields

$$
\left\{\begin{array}{l}
\mathcal{L}_{p} \varphi_{k(p)}=\frac{1}{k(p)} \mathcal{F}_{p} \varphi_{k(p)}, \\
\text { B. C.[p] on } \varphi_{k(p)}
\end{array}\right.
$$

where now the spectrum $\sigma_{p}[k]$ and the eigenmodes $\varphi_{k(p)}$ depend on the control parameter $p$. In this context, a natural question concerns the existence of a critical value of $p$, say $p=p_{c}$, for which the system is exactly critical, i.e., the dominant eigenvalue of Eq. (5) satisfies $k\left(p_{c}\right)=1$. Equations of this kind arise for instance when trying to determine the critical control rod position or the critical boron concentration for reactor cores. More broadly, several eigenvalue 
problems associated to the linear Boltzmann equation can be also formally recast in the form of Eq. (5): examples encompass the well-known $\alpha$-eigenvalue equation, where

$$
\mathcal{L}_{\alpha}=\mathcal{L}+\frac{\alpha}{v},
$$

the albedo eigenvalue equation, where

$$
\text { B. C. }[\beta]: \varphi_{k(\beta)}(\mathbf{x}, \mathbf{\Omega}, E)=\beta \varphi_{k(\beta)}\left(\mathbf{x}, \mathbf{\Omega}^{\prime}, E\right)
$$

on the boundary $\mathbf{x} \in \partial D$ of the core, with $\mathbf{n} \cdot \boldsymbol{\Omega}<0$ and $\boldsymbol{\Omega}^{\prime}=\boldsymbol{\Omega}-2(\mathbf{n} \cdot \boldsymbol{\Omega}) \mathbf{n}$, or the buckling eigenvalue equation, where

$$
\mathcal{L}_{B}=\mathcal{L}+i \mathbf{B} \cdot \boldsymbol{\Omega}
$$

for a fixed direction $\boldsymbol{\mu}=\mathbf{B} /|\mathbf{B}|$.

The critical value $p_{c}$ can be determined by a generalization of the power iteration method. The idea is that the power iteration yields $k\left(p_{c}\right)=1$ for the critical value $p_{c}$. Starting from a guess value $p^{(0)}$, the power iteration is applied and the corresponding fundamental eigenvalue $k\left(p^{(0)}\right)$ is computed. One needs then to introduce some update rule for $p^{(m+1)}$ at step $m$, based on the recorded values of $p^{(j)}$ and $k\left(p^{(j)}\right)$, for $j=0,1, \cdots, m$. For the sake of simplicity, we will assume here that the update rule is local (i.e., memoryless), and that it can be expressed as

$$
p^{(m+1)}=G\left(p^{(m)}, k\left(p^{(m)}\right)\right) .
$$

The functional form of $G$ is in principle arbitrary, although some constraints must be respected. In particular, we must have

$$
G(x, 1)=x,
$$

which means that at convergence the critical value $p_{c}$ must be a fixed point of the search algorithm. Another natural requirement on $G$ is that it should drive the parameter $p$ towards its critical value. For instance, if $k(p)>1$ and $\partial k(p) / \partial p>$ 0 , then we require that $G(p, k(p))<p$. This additional requirement on $G$ can be concisely summarized, for any possible combination of directions of inequalities, as

$$
\frac{G(p, k(p))-p}{k(p)-1} \frac{\partial k(p)}{\partial p}<0 .
$$

A common functional form for $G$ is a power law

$$
G(p, k)=p k^{\gamma},
$$


which automatically satisfies requirement (10). The second requirement, Eq. (11), imposes

$$
\gamma p \frac{\partial k(p)}{\partial p}<0,
$$

which can always be arranged by a suitable choice of the sign of $\gamma$.

We are thus led to consider the coupled dynamical system

$$
\left\{\begin{array}{l}
\mathcal{L}_{p^{(m)}} \varphi_{k\left(p^{(m)}\right)}^{(n+1)}=\frac{1}{k^{(n)}\left(p^{(m)}\right)} \mathcal{F}_{p^{(m)}} \varphi_{k\left(p^{(m)}\right)}^{(n)} \\
\text { B. C. }\left[p^{(m)}\right] \text { on } \varphi_{k\left(p^{(m)}\right)}^{(n)} \\
p^{(m+1)}=G\left(p^{(m)}, k\left(p^{(m)}\right)\right)
\end{array}\right.
$$

where the former equation is first solved for fixed control parameter $p^{(m)}$ by power iteration in order to determine $k^{(n)}\left(p^{(m)}\right) \stackrel{n}{\rightarrow} k\left(p^{(m)}\right)$ for large $n$ (the associated boundary conditions possibly also depending on $\left.p^{(m)}\right)$, and the latter equation is then used in order to determine the next value $p^{(m+1)}$ of the control parameter. For sufficiently large $m$, the dynamical system in Eq. (14) supposedly converges to the sought fixed point $p^{(m)} \stackrel{m}{\rightarrow} p_{c}$ and correspondingly $\varphi_{k\left(p^{(m)}\right)} \stackrel{m}{\rightarrow} \varphi_{k\left(p_{c}\right)}$, with $k\left(p^{(m)}\right) \stackrel{m}{\rightarrow} 1$.

Although formally emerging as an eigenvalue search problem, Eq. (14) has a clear real-life counterpart: due to an initial perturbation, the core may be driven into a super- or sub-critical state. By monitoring over some neutron generations the discrepancy between the resulting $k$ and unit, the control parameter is adjusted (either by an operator or by stabilizing physical feedbacks) in order to compensate the reactor criticality excess or deficit. Then, the reactor state is again monitored along some generations, and a new value of $k$ is determined. The procedure is iterated until $k$ is unit for some value $p=p_{c}$, and the reactor is stationary.

\section{Eigenvalue searches for the rod model}

For the sake of concreteness, the behaviour of critical-parameter searches is best illustrated on the rod model, which is possibly the simplest example of space- and direction-dependent transport problem (Wing, 1962; Montagnini and Pierpaoli, 1971): particles move at constant speed $v$ along a line (the rod) and undergo collision events with total cross section $\Sigma_{t}$. Because of the geometric constraints, only two directions of flight are allowed, namely forward $(\boldsymbol{\Omega}=+)$ 
and backward ( $\Omega=-)$; here, we furthermore assume that scattering and fission are isotropic, i.e., that directions taken by the particles after a collision are sampled with equal probability, and a single fissile isotope is present. If we define $\varphi_{k}^{+}(x)$ and $\varphi_{k}^{-}(x)$ to be the angular flux at position $x$ in the positive and negative direction, respectively, Eq. (1) takes the form of two coupled ordinary first-order differential equations

$$
\pm \frac{\partial}{\partial x} \varphi_{k}^{ \pm}(x)+\Sigma_{t} \varphi_{k}^{ \pm}(x)-\Sigma_{s} \varphi_{k}(x)=\frac{v_{f}}{k} \Sigma_{f} \varphi_{k}(x),
$$

where $\varphi_{k}(x)=\left[\varphi_{k}^{+}(x)+\varphi_{k}^{-}(x)\right] / 2$ is the scalar flux integrated over the directions. We will assume that the viable space is a segment $[0, L]$, for some positive $L$.

\subsection{Dispersion laws}

Suppose now that some control parameter $p$ is introduced in $\mathrm{Eq}(15)$, either in the operator or in the boundary conditions. We will denote by $\varphi_{k(p)}^{ \pm}(x)$ the solutions of the rod model equations including the effects of the control parameter $p$. We would like to determine the corresponding dispersion law $\Lambda(p, k)$, whose zeros provide the spectrum $\sigma_{p}[k]$ for a given value of $p$. Conversely, the roots of $\Lambda(p, k)=0$, solved as a function of $p$ for $k(p)=1$, are the values of the control parameter $p$ for which the system is critical.

The general integrals of Eq. 15 are easily obtained as linear combinations of exponential functions up to two integration constants, say $\kappa_{1}$ and $\kappa_{2}$, that must be fixed by the boundary conditions. In the usual case, the boundary conditions translate into a system of linear equations for $\kappa_{1}$ and $\kappa_{2}$ with coefficients given by a $2 \times 2$ matrix $M(p, k)$. The existence of non-trivial solutions requires

$$
\Lambda(p, k)=\operatorname{det} M(p, k)=0
$$

which is the sought dispersion relation. This is an implicit equation for the eigenvalue $k$, the control parameter $p$ and the physical constants (the cross sections, the average fission yield, and the domain size).

\subsection{Reactor period search}

A prominent example of eigenvalue problem that can be solved by a modified power iteration is the so-called alpha eigenvalue equation, where the dominant eigenvalue physically represents the inverse of the asymptotic reactor period (Weinberg, 1952). This class of problems has attracted intensive research efforts, on both the theoretical (Larsen and Zweifel, 1974; Azmy and Sartori, 2010) and computational side (Brockway et al., 1985, Hill, 1983; Nolen et al. 
2012), especially in view of reactor period (Zoia et al., 2015; Nauchi, 2014) and neutron die-away constants (Zoia et al., 2014; Ye et al., 2006, Yamamoto and Miyoshi, 2003) calculations. The $\alpha$-eigenvalue equation for the rod model reads

$$
\pm \frac{\partial}{\partial x} \varphi_{k(\alpha)}^{ \pm}(x)+\frac{\alpha}{v \Sigma_{t}} \varphi_{k(\alpha)}^{ \pm}(x)+\Sigma_{t} \varphi_{k(\alpha)}^{ \pm}(x)-\Sigma_{s} \varphi_{k(\alpha)}(x)=\frac{v_{f}}{k(\alpha)} \Sigma_{f} \varphi_{k(\alpha)}(x),
$$

with leakage boundary conditions $\varphi_{k(\alpha)}^{+}(0)=0$ and $\varphi_{k(\alpha)}^{-}(L)=0$. Eq. (16) yields the dispersion law

$$
\Lambda(\alpha, k)=\cosh \left(L \Sigma_{t} \sqrt{Q_{\alpha}}\right)+\frac{\left(\frac{\alpha}{v \Sigma_{t}}+1-\frac{c_{k}}{2}\right) \sinh \left(L \Sigma_{t} \sqrt{Q_{\alpha}}\right)}{\sqrt{Q_{\alpha}}},
$$

where we have defined

$$
Q_{\alpha}=\left(\frac{\alpha}{v \Sigma_{t}}+1\right)\left(\frac{\alpha}{v \Sigma_{t}}+1-c_{k}\right)
$$

and

$$
c_{k}=\frac{\Sigma_{s}+\frac{v \Sigma_{f}}{k}}{\Sigma_{t}}
$$

which represents the average number of secondary particles per collision, normalized to $k$. The associated spectrum, i.e., the roots of $\Lambda(\alpha, k)=0$ in Eq. (18), has a rather intricate structure, which has been extensively examined by several authors (see, e.g., Larsen and Zweifel (1974); Montagnini and Pierpaoli (1971)). In particular, it has been shown that, for $k(\alpha)=1$, Eq. (18) has a finite number of real eigenvalues $\alpha$, plus a countable infinity of complex eigenvalues associated to oscillating modes (Montagnini and Pierpaoli, 1971).

For very large systems, $L \Sigma_{t} \gg 1$, the roots of Eq. (18) become independent of $L$ and there exists only one eigenvalue satisfying the simpler relation

$$
\alpha=v \Sigma_{t}\left(c_{k}-1\right)
$$

Correspondingly, the $k$-eigenvalue for a given $\alpha$ reads

$$
k(\alpha)=\frac{k_{\infty}}{1+\alpha / \alpha_{*}}
$$

where $k_{\infty}=v \Sigma_{f} /\left(\Sigma_{t}-\Sigma_{s}\right)$ is the fundamental $k$-eigenvalue of the system with $\alpha=0$ and $\alpha_{*}=v\left(\Sigma_{t}-\Sigma_{s}\right)$ is the so-called removal rate, i.e., the frequency at which particles are removed from the system because of captures. Eqs. (3.2) are physically meaningful only for $\alpha>-\alpha_{*}$. 
The asymptotic reactor period is determined in closed form by setting $k(\alpha)=$ 1 in Eq. (21):

$$
\alpha=\alpha_{*}\left(k_{\infty}-1\right) .
$$

However, for the sake of illustrating critical parameter searches, we pretend that an analytical solution is not available and that a solution must be numerically sought, as detailed in Sec. 2. We select Eq. (12) as an update rule for $\alpha$. The sign of $\gamma$ must be fixed according to Eq. (13): since $\partial k(\alpha) / \partial \alpha<0$ for Eq. (17), $\gamma$ and $\alpha$ must have the same sign. For simplicity, we take $\gamma=1$ for $\alpha \geq 0$ and $\gamma=-1$ for $\alpha<0$.

With these provisions, the update rule for $\alpha$ reads

$$
\alpha^{(m+1)}= \begin{cases}\alpha^{(m)} \cdot k\left(\alpha^{(m)}\right) & \text { if } k_{\infty} \geq 1 \\ \frac{\alpha^{(m)}}{k\left(\alpha^{(m)}\right)} & \text { if } k_{\infty}<1 .\end{cases}
$$

We draw the attention of the reader to the fact that Eq. (12) preserves the sign of $\alpha$ (which might be convenient for applications involving Monte Carlo power iteration); therefore, the sign of the initial value $\alpha^{(0)}$ must be the correct one, i.e., the same as the dominant $\alpha$ eigenvalue. This imposes the restriction $\alpha^{(0)}>0$ for $k_{\infty}>1$, and $-\alpha_{*}<\alpha^{(0)}<0$ for $k_{\infty}<1$. In the general case, one would numerically solve for $k\left(\alpha^{(0)}\right)$ by applying the power iteration to Eq. (17); the update rule Eq. (24) is then applied and a new value $\alpha^{(1)}$ is determined. The procedure is in principle iterated until convergence.

However, does convergence ever occur? For the problem at hand, we can simply substitute Eq. (22) (which replaces the iterative search for the dominant $k$-eigenvalue for a given $\alpha$ ) in Eq. (24) to explicitly obtain the actual update rule

$$
\alpha^{(m+1)}= \begin{cases}k_{\infty} \frac{\alpha^{(m)}}{1+\alpha^{(m)} / \alpha_{*}} & \text { if } k_{\infty} \geq 1 \\ \frac{1}{k_{\infty}} \alpha^{(m)}\left(1+\alpha^{(m)} / \alpha_{*}\right) & \text { if } k_{\infty}<1 .\end{cases}
$$

This update rule clearly admits $\alpha=0$ and $\alpha=\alpha_{*}\left(k_{\infty}-1\right)$ as fixed points, whose stability depends on the absolute value of the derivative of the map evaluated at the fixed points. If such value is smaller than one, then the fixed point is stable; otherwise, it is unstable. It is easy to verify that $\alpha=0$ is always unstable, for any $k_{\infty}$. The stability of $\alpha=\alpha_{*}\left(k_{\infty}-1\right)$ is always ensured for the map corresponding to $k_{\infty} \geq 1$. For the map corresponding to $k_{\infty}<1, \alpha=\alpha_{*}\left(k_{\infty}-1\right)$ is stable for $1>k_{\infty}>1 / 3$, and becomes unstable for smaller values of $k_{\infty}$. The precise 

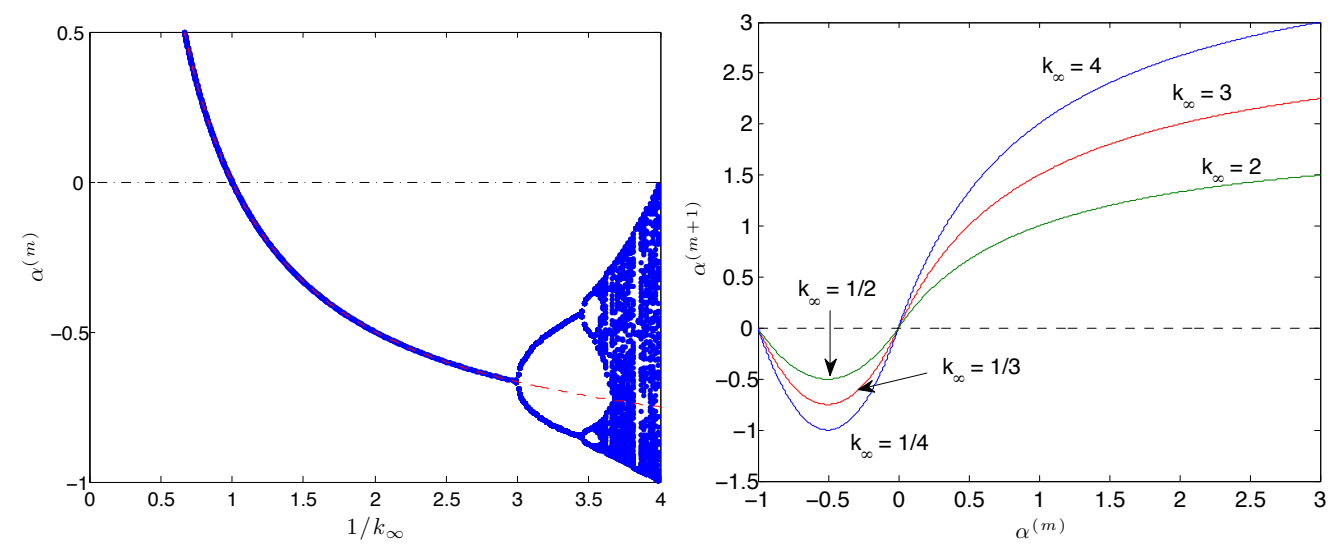

Figure 1: Left. Application of the iterated map for $\alpha^{(m)}$ as a function of $1 / k_{\infty}$, for $\alpha_{*}=1$. For $k_{\infty}>1 / 3$, the eigenvalue search (displayed as blue points) finds the exact solution (displayed as a red dashed line). For $k_{\infty}<1 / 3$, the map undergoes a series of period-doubling bifurcations, and the search for the dominant eigenvalue fails. Right. The map given in Eq. 25] as a function of $1 / k_{\infty}$, for $\alpha_{*}=1$.

behaviour of the nonlinear map in Eq. 25) for the case $k_{\infty}<1$ can be determined by remarking that this update rule is conjugate to the celebrated logistic map

$$
f_{a}(y)=a y(1-y)
$$

under the variable transformation

$$
y^{(m)}=-\frac{\alpha^{(m)}}{\alpha_{*}},
$$

and $a=1 / k_{\infty}$. We can immediately deduce the key features of Eq. (25) from those of the logistic map, which has attracted intensive research efforts (May, 1976; Collet and Eckmann, 1980; Feigenbaum, 1983). For $1>k_{\infty}>1 / 2$, the map will converge to $\alpha_{*}\left(k_{\infty}-1\right)$, for almost all initial guesses. For $1 / 2>$ $k_{\infty}>1 / 3$, the map will also eventually approach the same value through damped oscillations around that value. The rate of convergence in this interval is linear, except for $k_{\infty}=1 / 3$, when it becomes slower than linear. When $1 / 3>$ $k_{\infty}>1 /(1+\sqrt{6}) \simeq 0.2899$, from almost all initial conditions the algorithm will approach permanent oscillations between two values: technically speaking, the map has undergone a period-doubling bifurcation (May, 1976, Collet and Eckmann, 1980; Feigenbaum, 1983). For $0.2899>k_{\infty}>0.2822$, from almost all initial conditions the map will permanently oscillate among four values. By further decreasing $k_{\infty}$, the map will display oscillations among 8 values, then 16 , 
and so on. This behaviour is known as a period-doubling cascade, and is basically due to the fact that $i$ ) the map has a single (quadratic) maximum in the region $k_{\infty}<1$, and ii) this maximum is an increasing function of the control parameter $1 / k_{\infty}$ (Feigenbaum, 1978, 1979, 1983). This is illustrated in Fig. 1 . The absence of a maximum for $k_{\infty}>1$ is responsible for the stability of the fixed point for the eigenvalue search in the super-critical regime. The lengths of the $k_{\infty}$ intervals that yield oscillations of a given length decrease for increasing period doubling order $q$; for large $q$, the ratio between the lengths of two successive bifurcation intervals eventually approaches the so-called Feigenbaum constant (Feigenbaum, 1983)

$$
\delta=\lim _{q \rightarrow \infty} \frac{\left(1 / k_{\infty}\right)_{q-1}-\left(1 / k_{\infty}\right)_{q-2}}{\left(1 / k_{\infty}\right)_{q}-\left(1 / k_{\infty}\right)_{q-1}}=4.669 \ldots
$$

The value $k_{\infty} \simeq 0.2801$ corresponds to the end of the period-doubling cascade and the onset of a chaotic behaviour for the map: from almost all initial conditions, slight variations in the initial guess will induce exceedingly different results, and the oscillations will have an infinite period. For most values below $k_{\infty} \simeq 0.2801$ the map will display chaos; however, some isolated ranges of $k_{\infty}$ (which take the name of islands of stability) display non-chaotic behaviour (Collet and Eckmann, 1980).

As far as the search of the dominant $\alpha$ eigenvalue is concerned, the important point of this analysis is that the iteration of the map given in Eq. 25) will basically fail whenever $k_{\infty}<1 / 3$, i.e., after the first period-doubling bifurcation. For sub-critical systems beyond this threshold value of $k_{\infty}$, the search of the dominant $\alpha$ eigenvalue will eventually lock on a cycle oscillating between two values, none of which corresponds to the sought solution $\alpha=\alpha_{*}\left(k_{\infty}-1\right)$. For even more sub-critical configurations, the situation worsens, and the map oscillates between more and more pairs of values, until a chaotic aperiodic behaviour emerges. For $k_{\infty}=1 / 4$, the map codomain spans the entire allowed interval $\left[-\alpha_{*}, 0\right]$, and for $k_{\infty}<1 / 4$ almost any initial condition taken in $\left[-\alpha_{*}, 0\right]$ will escape to infinity. the eigenvalue search algorithm will eventually crash. This behaviour is illustrated in Fig. 1 .

For finite domain size $L$, based on the analysis above it would be tempting to postulate again a functional form of the kind

$$
k(\alpha)=\frac{k}{1+\alpha / \alpha_{*}},
$$

where $k$ is the dominant eigenvalue of the $k$-eigenvalue equation (15), with leakage boundary conditions, and $\alpha_{*}$ is a problem-dependent removal rate that has to 
be determined. Remark that the $k(\alpha)$ parameter for finite-size systems cannot be derived analytically from the dispersion law $\Lambda(\alpha, k)=0$, and must be found by applying the power iteration scheme described in the previous section. Actually, by taking the $\alpha$-eigenvalue equation (17) and applying the term-by-term scalar product with the adjoint eigenfunction $\varphi_{k}$ of the $k$-eigenvalue equation (15), and by taking the $k$-eigenvalue equation and applying the term-by-term scalar product with the adjoint eigenfunction $\varphi_{k(\alpha)}^{\dagger}$ of the $\alpha$-eigenvalue equation (17), we get precisely Eq. (29), with

$$
\alpha_{*}=\frac{\left\langle\varphi_{k(\alpha)}^{\dagger}, \mathcal{L} \varphi_{k}\right\rangle}{\left\langle\varphi_{k(\alpha)}^{\dagger}, \frac{1}{v} \varphi_{k}\right\rangle},
$$

where $\langle\cdot, \cdot\rangle$ denotes the scalar product, $\dagger$ stands for adjoint, and $\mathcal{L}$ is the net disappearance operator. The term $\alpha_{*}$ is thus an adjoint-weighted bi-linear form that can be interpreted as the removal rate for finite-size systems. For large $L \Sigma_{t}$, it is easy to show that $\alpha_{*} \rightarrow v\left(\Sigma_{t}-\Sigma_{s}\right)$, since the flux eigenfunctions are spatially flat. Equation (29) establishes a correspondence between the $k$ eigenvalues and the $\alpha$ eigenvalues by means of the term $\alpha_{*}$, which depends on both the $k(\alpha)$ - and $k$-eigenfunctions.

Because of $\alpha_{*}$, the relation in Eq. (29) will display a dependence on $\alpha$ and $k$ that is more involved than the one for the infinite system. However, numerical simulations show that $\alpha_{*}$ only weakly depends on $\alpha$ (provided that the removal rate is dominated by captures rather than by leakage), so that the chaotic behaviour observed for the case of the eigenvalue search in infinite-size systems is qualitatively carried over to finite-size systems. In particular, we can still apply the change of variables given in Eq. (27), which transforms the update rule for finite $L$ into the logistic map. In order to substantiate our claim, we have performed a series of eigenvalue searches in finite-size rod models: the results are reported in Fig. 2. It is apparent that the eigenvalue search suffers from the same issue affecting infinite systems: in particular, for deep sub-critical systems the search will undergo a series of period-doubling bifurcations that will end up in the failure of the algorithm. When $k>1$, the update map is a monotonous function of $\alpha$, and the power iteration will converge to the exact solution.

\subsection{Buckling eigenvalue equation}

Buckling eigenvalue equations emerge for reactor physics calculations by assuming that the neutron flux can be factorized into a form of the kind $\varphi_{k}^{ \pm}(x)=$ $e^{i \mathbf{B} \cdot \mathbf{x}} \varphi_{k(B)}^{ \pm}(x)$, representing the product of a lattice structure factor $\exp (i \mathbf{B} \cdot \mathbf{x})$ and a fine structure factor $\varphi_{k(B)}^{ \pm}(x)$. Buckling eigenvalues emerge for instance in the 


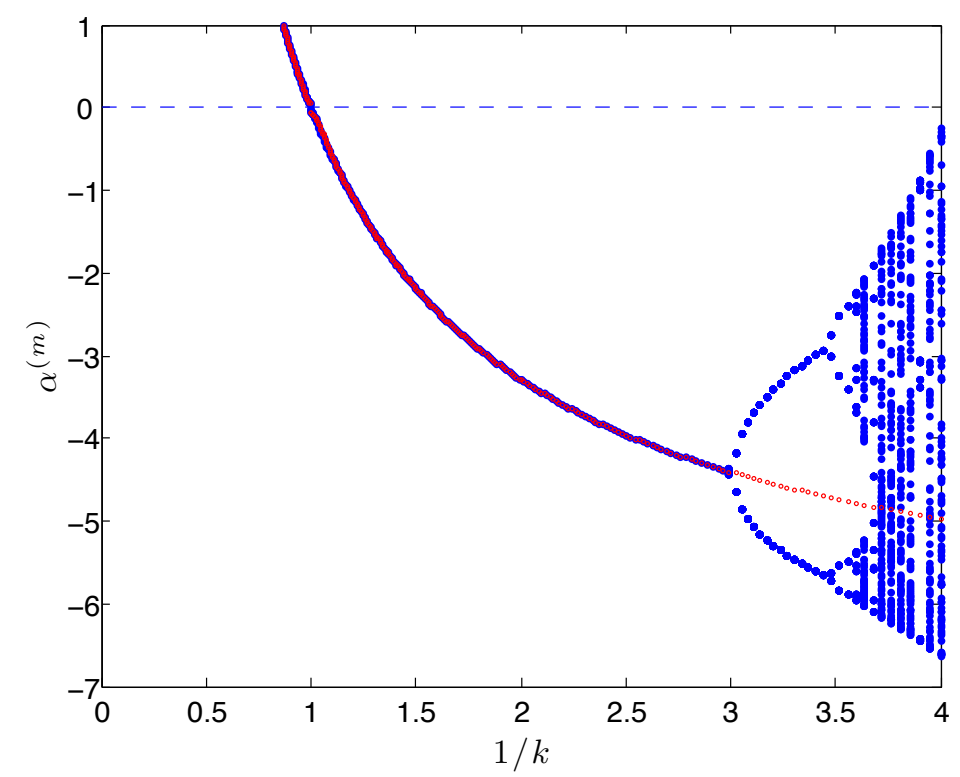

Figure 2: Application of the iterated map for $\alpha^{(m)}$ as a function of $1 / k$. For $k>1 / 3$, the eigenvalue search (displayed as blue points) finds a single solution. For $k<1 / 3$, the map undergoes a series of period-doubling bifurcations, and the search for the dominant eigenvalue fails. The true dominant $\alpha$ eigenvalue has been determined numerically by setting $\gamma=-1 / 2$ in the update rule and is displayed as red dots.

treatment of fundamental-mode neutron leakage in the presence of voids (Gelbard and Lell, 1976) and have also inspired recent work concerning complexweight Monte Carlo schemes (Yamamoto, 2012a b; Rouchon, 2017).

For the rod model, only the positive or negative directions are allowed: by taking $\mathbf{B}$ oriented in the positive direction $\boldsymbol{\Omega}=+$, the resulting eigenvalue equations read

$$
\pm\left(\frac{\partial}{\partial x}+i B\right) \varphi_{k(B)}^{ \pm}(x)+\Sigma_{t} \varphi_{k(B)}^{ \pm}(x)-\Sigma_{s} \varphi_{k(B)}(x)=\frac{v_{f}}{k(B)} \Sigma_{f} \varphi_{k(B)}(x),
$$

with periodic boundary conditions $\varphi_{k(B)}^{+}(0)=\varphi_{k(B)}^{+}(L)$ and $\varphi_{k(B)}^{-}(0)=\varphi_{k(B)}^{-}(L)$. By applying the same strategy as for $\alpha$ eigenvalues, we are led to the following relation

$$
k(B)=\frac{k}{1+\frac{\left\langle\varphi_{k(B)}^{\dagger}, i \mathbf{\Omega} \cdot \mathbf{B} \varphi_{k}\right\rangle}{\left\langle\varphi_{k(B)}^{\dagger}, \mathcal{L} \varphi_{k}\right\rangle}},
$$

whose structure is very similar to that of Eq. (29). An explicit expression for $k(B)$ can be obtained by observing that the dispersion law for the buckling eigenvalues 
reads

$$
\Lambda(B, k)=\cos (L B)-\cos \left(L \Sigma_{t} \sqrt{c_{k}-1}\right) .
$$

The equation $\Lambda(B, k)=0$ admits the following analytical solution:

$$
k(B)=\frac{k_{\infty}}{1+B^{2} / B_{*}^{2}},
$$

where again $k_{\infty}=v \Sigma_{f} /\left(\Sigma_{t}-\Sigma_{s}\right)$ is the fundamental eigenvalue of the system with $B=0$, and $B_{*}^{2}=\Sigma_{t}\left(\Sigma_{t}-\Sigma_{s}\right)$ is the maximum allowed buckling. Actually, it can be shown that $B_{*}^{-2}$ is proportional to the adjoint-weighted migration area of the neutrons in the system (Gelbard and Lell, 1976).

Eq. (34) is formally similar to Eq. (22). Indeed, the relevant update rule for the buckling parameter reads

$$
\left(B^{2}\right)^{(m+1)}= \begin{cases}k_{\infty} \frac{\left(B^{2}\right)^{(m)}}{1+\left(B^{2}\right)^{(m)} / B_{*}^{2}} & \text { if } k_{\infty} \geq 1 \\ \frac{1}{k_{\infty}}\left(B^{2}\right)^{(m)}\left[1+\left(B^{2}\right)^{(m)} / B_{*}^{2}\right] & \text { if } k_{\infty}<1 .\end{cases}
$$

The sub-critical case of Eq. (35) can also be shown to be conjugate to the logistic map with the variable transformation

$$
y=-\frac{B^{2}}{B_{*}^{2}} .
$$

The logistic map parameter is given by $a=1 / k_{\infty}$. We thus expect the buckling eigenvalue search to display the same behaviour as the $\alpha$ eigenvalue search: for super-critical systems, the search algorithm by power iteration is stable and converges to the fundamental buckling eigenvalue

$$
B^{2}=B_{*}^{2}\left(k_{\infty}-1\right) \text {. }
$$

When on the contrary the system is sub-critical, for $k_{\infty}<1 / 3$ the power iteration will undergo a series of period-doubling bifurcations, and the search will fail.

Observe that the update rule for the $\alpha$ eigenvalue search was a linear function of the dimensionless ratio $\alpha / \alpha_{*}$, whereas for the buckling the update rule depends quadratically on the ratio $B / B_{*}$. Except for this minor change, the buckling eigenvalue search will display exactly the same behaviour as the $\alpha$ eigenvalue search in infinite systems. 
It is legitimate to ask whether using a different update rule would similarly lead to the failure of the search algorithm. For sub-critical configurations, it is convenient to substitute $i B \rightarrow B$ and rewrite Eq. (38) as

$$
\pm\left(\frac{\partial}{\partial x}+B\right) \varphi_{k(B)}^{ \pm}(x)+\Sigma_{t} \varphi_{k(B)}^{ \pm}(x)-\Sigma_{s} \varphi_{k(B)}(x)=\frac{v_{f}}{k(B)} \Sigma_{f} \varphi_{k(B)}(x),
$$

with the update rule

$$
B^{(m+1)}=\frac{B^{(m)}}{k\left(B^{(m)}\right)} .
$$

Numerical simulations show that this update rule also exhibits period-doubling bifurcations in the sub-critical regime, albeit at different values of $k_{\infty}$. This can be understood as a consequence of the functional shape of the update map, which displays a single quadratic maximum for $k_{\infty}<1$ and thus belongs to the class of universality of the logistic map (although not necessarily by conjugation).

\subsection{Albedo eigenvalue equation}

The critical albedo search consists in imposing albedo conditions on the boundaries (or part of the boundaries) of the system, and determining the albedo value $\beta$ (if any) that makes the reactor critical. Such problems emerge for instance in connection with Monte Carlo depletion calculations at the lattice scale, as a way of reproducing whole-core effects at the boundaries of the assembly (Cho et al., 2009; Yun and Cho, 2009). For the albedo search, the eigenvalue equation for the rod model is left unchanged with respect to the case of the regular $k$-eigenvalue equation, namely,

$$
\pm \frac{\partial}{\partial x} \varphi_{k(\beta)}^{ \pm}(x)+\Sigma_{t} \varphi_{k(\beta)}^{ \pm}(x)-\Sigma_{s} \varphi_{k(\beta)}(x)=\frac{v_{f}}{k(\beta)} \Sigma_{f} \varphi_{k(\beta)}(x) .
$$

However, the eigenvalue $\beta$ appears in the albedo boundary conditions $\varphi_{k(\beta)}^{+}(0)=$ $\beta \varphi_{k(\beta)}^{-}(0)$ and $\varphi_{k(\beta)}^{-}(L)=\beta \varphi_{k(\beta)}^{+}(L)$. For the sake of simplicity, we will assume that the albedo condition is imposed at both ends of the domain $[0, L]$. The smallest admissible albedo value is $\beta=0$, which corresponds to perfect leakage boundary conditions: thus, for sufficiently super-critical systems, no albedo boundary condition $\beta>0$ can make the system critical.

The relation between $k(\beta)$ and $k$ (the latter being the dominant eigenvalue of the equations with $\beta=0$ ) can again be expressed by resorting to the scalar products between direct and adjoint eigenfunctions, and reads

$$
k(\beta)=\frac{k}{1-\frac{\Delta(\beta)}{16^{\left\langle\varphi_{k(\beta)}^{\dagger}, \mathcal{L} \varphi_{k}\right\rangle}},}
$$


where the term $\Delta(\beta)=\left\langle\varphi_{k(\beta)}^{\dagger}, \nabla \cdot \boldsymbol{\Omega} \varphi_{k}\right\rangle+\left\langle\nabla \cdot \boldsymbol{\Omega} \varphi_{k(\beta)}^{\dagger}, \varphi_{k}\right\rangle$ does not vanish because of the different boundary conditions on Eqs. (15) and (40). Actually, by the Gauss theorem, the term $\Delta(\beta)$ can be rewritten as

$$
\Delta(\beta)=\int_{S} d S \mathbf{n} \cdot \int d \boldsymbol{\Omega} \varphi_{k(\beta)}^{\dagger} \mathbf{\Omega} \varphi_{k}=\beta \int_{S} d S \mathbf{n} \cdot \int_{\text {out }} d \mathbf{\Omega} \varphi_{k(\beta)}^{\dagger}(\mathbf{\Omega}-\mathbf{n} \cdot \mathbf{\Omega} \mathbf{\Omega}) \mathbf{J}_{k}(\mathbf{\Omega}),
$$

where we have used the albedo boundary conditions. Here $\mathbf{J}_{k}(\boldsymbol{\Omega})=\boldsymbol{\Omega} \varphi_{k}(\boldsymbol{\Omega})$ denotes the neutron vector current. The quantity $\Delta(\beta) / \beta$ thus physically represents the out-going neutron current weighted by the in-going adjoint neutron flux. By analogy with the case of $\alpha$ and buckling eigenvalues, it is convenient to define

$$
\beta_{*}=\frac{\left\langle\varphi_{k(\beta)}^{\dagger}, \mathcal{L} \varphi_{k}\right\rangle}{\int_{S} d S \mathbf{n} \cdot \int_{\text {out }} d \boldsymbol{\Omega} \varphi_{k(\beta)}^{\dagger}(\mathbf{\Omega}-\mathbf{n} \cdot \mathbf{\Omega} \mathbf{\Omega}) \mathbf{J}_{k}(\mathbf{\Omega})},
$$

so that we can formally rewrite Eq. (41) as

$$
k(\beta)=\frac{k}{1-\beta / \beta_{*}} .
$$

The dispersion law for the albedo eigenvalues reads

$$
\begin{aligned}
\Lambda(\beta, k) & =\left(\beta^{2}-1\right) \cosh \left(L \Sigma_{t} \sqrt{1-c_{k}}\right) \\
& +\frac{1}{2} \frac{c_{k}(1+\beta)^{2}-1-\beta^{2}}{\sqrt{1-c_{k}}} \sinh \left(L \Sigma_{t} \sqrt{1-c_{k}}\right) .
\end{aligned}
$$

The parameter $\beta$ that solves $\Lambda(\beta, k)=0$ can be determined analytically, yielding

$$
\beta=\frac{1-Q_{\beta}}{1+Q_{\beta}}
$$

where

$$
Q_{\beta}=\sqrt{\left(\frac{k_{\infty}}{k(\beta)}-1\right) \frac{\Sigma_{a}}{\Sigma_{t}}} \tan \left(\frac{L \Sigma_{t}}{2} \sqrt{\left.\left(\frac{k_{\infty}}{k(\beta)}-1\right) \frac{\Sigma_{a}}{\Sigma_{t}}\right)},\right.
$$

$\Sigma_{a}=\Sigma_{t}-\Sigma_{s}$ is the absorption cross section, and $k_{\infty}$ is defined as above. In particular, setting $k(\beta)=1$ in Eq. (47) yields the dominant albedo eigenvalue.

We have probed the eigenvalue search by solving Eq. (40) by power iteration, together with the update rule

$$
\beta^{(m+1)}=\frac{\beta^{(m)}}{17^{\left(\beta^{(m)}\right)}} .
$$




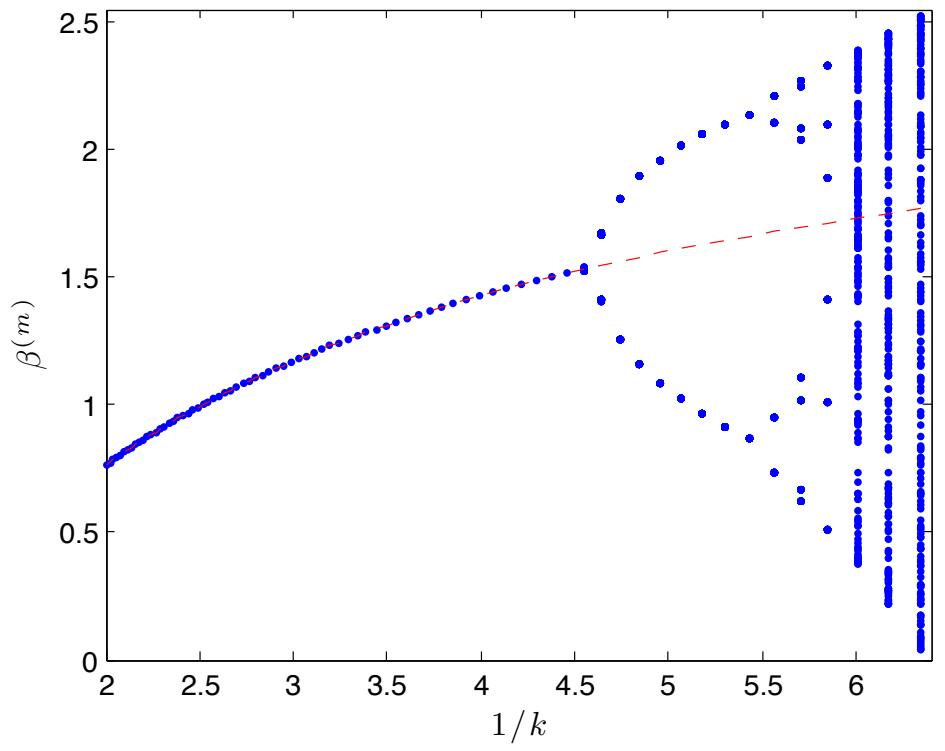

Figure 3: Application of the iterated map for $\beta^{(m)}$ as a function of $1 / k$. For sufficiently sub-critical systems, the map undergoes a series of period-doubling bifurcations, and the search for the dominant eigenvalue fails. The exact solution for the dominant eigenvalue as given by Eq. (47) is displayed as a red dashed line.

This is numerically illustrated in Fig. 3, where we compare the dominant eigenvalue found by the search algorithm with the exact result given in Eq. (47): for deep sub-critical systems, the albedo eigenvalue search undergoes period doubling, then exhibits chaos and ultimately fails. This can again be understood by observing that Eq. (49) is conjugate to the logistic map when setting $y=\beta / \beta_{*}$, provided that the quantity $\beta_{*}$ is a sufficiently slowly varying function of $\beta$.

\section{Generalization: the appearance of chaos in eigenvalue searches}

The findings described in the previous section by resorting to the rod model are not restricted to mono-energetic transport, and actually emerge in real-life applications. In order to illustrate this point, we present now an an example concerning the albedo eigenvalue search. We have recently implemented in the continuous-energy production Monte Carlo code TrIPOLI- $4{ }^{\circledR}$ (developed at CEA (Brun et al., 2015)) a new method for the determination of the albedo boundary conditions that make a multiplying system exactly critical: this functionality can be used for instance in the analysis of fuel depletion for assembly calculations (Yun and Cho, 2009; Cho et al., 2009). The albedo eigenvalue 


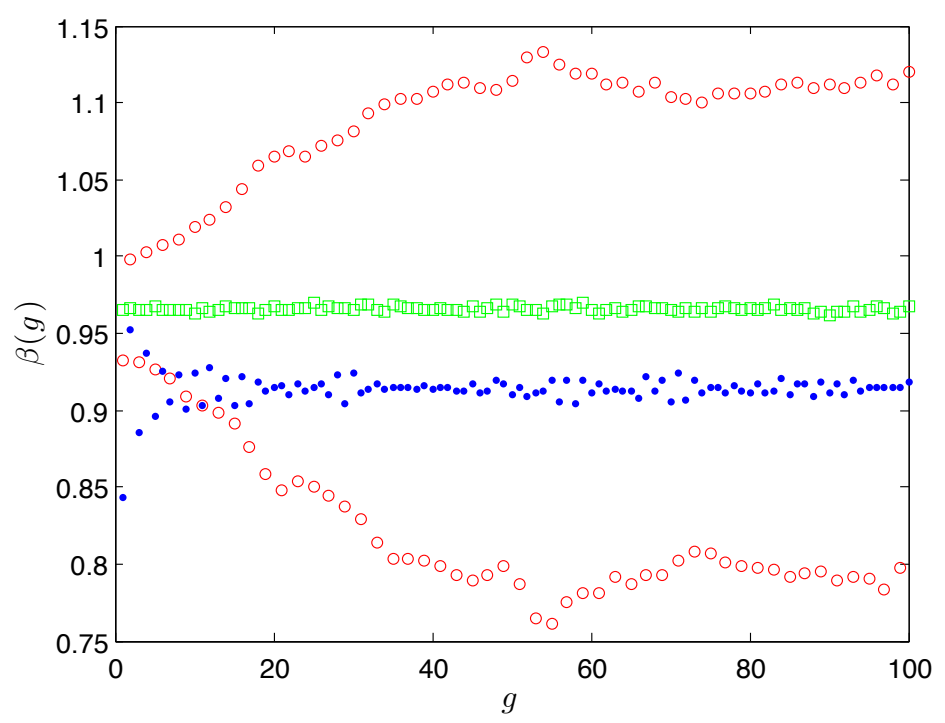

Figure 4: Albedo eigenvalue search with TRIPOLI-4 ${ }^{\circledR}$, for a UOX assembly. For beginning-oflife conditions, the assembly is slightly super-critical and the search algorithm converges to the sought eigenvalue as a function of the power iteration generations $g$ (blue dots). At higher burnup, the assembly has a lower multiplication factor, and the albedo search undergoes a perioddoubling bifurcation, leading to an oscillating series (red circles). This latter configuration has been re-run by setting $\gamma=-1 / 2$ : in this case, the convergence towards the sought albedo eigenvalue is ensured (green squares).

search has been implemented by modifying the standard power iteration of criticality calculations, including $\beta$ as an adjustable parameter: the albedo value is iteratively computed based on the $k$ value determined by the underlying power iteration.

For our calculations, we have chosen a three-dimensional model of UOX assembly with $17 \times 17$ fuel pin-cells. The albedo boundary condition is imposed on the radial surfaces of the assembly. At beginning-of-life conditions, the assembly is slightly super-critical, and the albedo search converges to the sought eigenvalue (see Fig. 4, where simulation results are displayed as a function of the power iteration generations). As the fuel burn-up increases and the effective multiplication factor decreases, the albedo search encounters the convergence issues described above for the rod model. In particular, for sufficiently low multiplication factor the search algorithm will bifurcate and lead to an oscillating series as a function of the power iteration generations (see Fig. 4). The presence of the statical noise intrinsically due to Monte Carlo simulation does not qualitatively alter the conclusions reached in the previous sections by using a deterministic 
power iteration.

Thus, the emergence of bifurcation instabilities in eigenvalue searches is not specific to mono-energetic or one-dimensional transport. Unfortunately, a rigorous characterization of the conditions for bifurcation of the eigenvalue search has eluded us; however, we have some plausibility arguments. Consider an eigenvalue search, and let $p$ be the eigenvalue. For a fixed value of $p$, the eigenvalue equation is actually a $k$-eigenvalue equation that may or may not admit a solution. In all the problematic cases discussed above, instabilities emerged when the update rule is taken to be

$$
p^{(m+1)}=\frac{p^{(m)}}{k\left(p^{(m)}\right)} .
$$

A trivial root for this equation is $p^{(m)}=0$. Moreover, if there is a finite value of $p$ (say $\bar{p}>0$; the argument is similar for $\bar{p}<0$ ) for which the corresponding multiplication factor $k(p)$ diverges to infinity, then this leads to a second root. Observe that the non-trivial root $\bar{p}$ can be formally seen as the dominant eigenvalue of the Boltzmann Eq. (5) when the fission operator is suppressed.

Specifically, for the examples shown in Sec. 3 for the rod model, the nontrivial roots of the update map exist and read

$$
\bar{\alpha}=-\alpha_{*}=-v\left(\Sigma_{t}-\Sigma_{s}\right)
$$

for $\alpha$ eigenvalue search in infinite systems,

$$
\bar{B}=B_{*}=\sqrt{\Sigma_{t}\left(\Sigma_{t}-\Sigma_{s}\right)}
$$

for buckling eigenvalue search, and

$$
\bar{\beta}=\frac{1-Q_{\infty}}{1+Q_{\infty}}
$$

for albedo eigenvalue search, with

$$
Q_{\infty}=-\sqrt{\frac{\Sigma_{a}}{\Sigma_{t}}} \tanh \left(\frac{L \Sigma_{t}}{2} \sqrt{\frac{\Sigma_{a}}{\Sigma_{t}}}\right) .
$$

455 The update rule Eq. (50) thus maps both $p^{(m)}=0$ and $p^{(m)}=\bar{p}$ on $p^{(m+1)}=$ 0 . Since the update rule is a smooth, non-trivial function of $p$, and since it preserves the sign of $p$, it must have at least one maximum for $0<p<\bar{p}$. If the maximum is unique, it will generally be quadratic; therefore, the update rule will typically satisfy the topological conditions for the emergence of chaos identified by Feigenbaum (Feigenbaum, 1978, 1979). This argument suggests that the route to instabilities for eigenvalue searches in sub-critical systems might be universal, and possibly manifest itself quite often. 


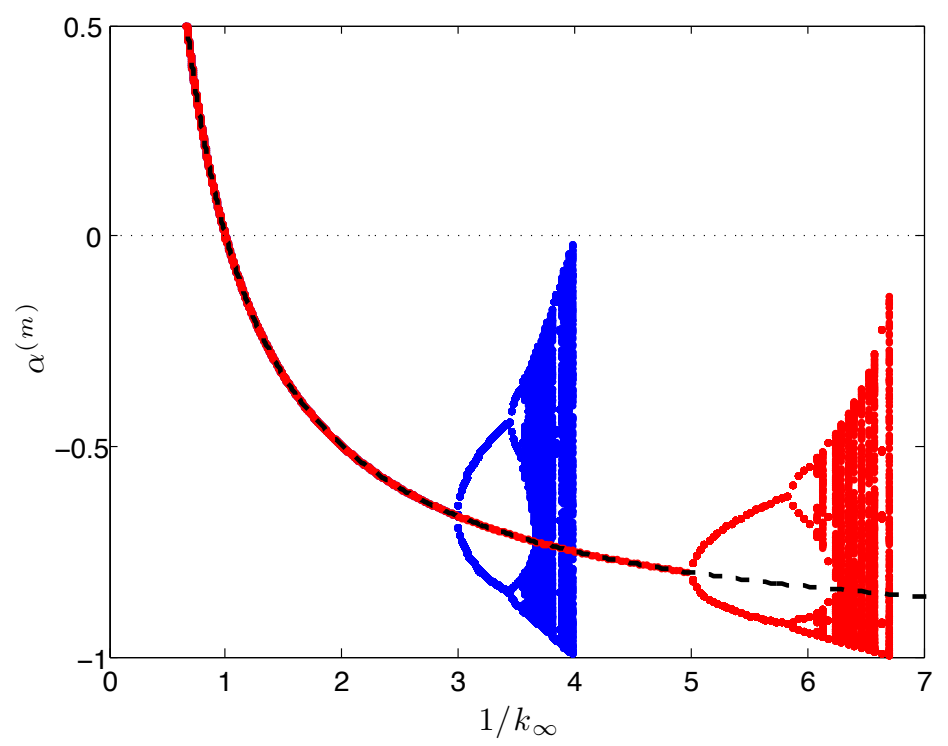

Figure 5: Bifurcation diagram for the sub-critical $\alpha$ eigenvalue search with $\gamma=-1$ (blue) and $\gamma=-1 / 2$ (red). The search with $\gamma=-1 / 2$ is able to converge also for the interval $3<1 / k_{\infty}<5$, where the search with $\gamma=-1$ fails. However, bifurcations still appear for larger values of $1 / k_{\infty}$. The black dashed line corresponds to the exact solution as given by Eq. 23.

\section{Possible workarounds for the instabilities}

We have shown so far that eigenvalue searches may fail to converge to the correct solution even in situations where the latter exists and represents a fixed point of the iterated search algorithm. Naturally, one may ask whether it is possible to recast the search problem in a way that ensures convergence, or at least mitigates the problems described above. This section discusses a few possible workarounds.

In all the cases described in Section 3 , the success of the search ultimately depends on the stability of the critical value of the parameter under iteration of the update map; in other words, iterated application of the update map on values close to the solution must converge to the solution. The stability of the update map with respect to infinitesimal perturbations is governed by the Jacobian of 
the map, calculated at the fixed point. For Eq. (12), this reads

$$
\begin{aligned}
\left.\frac{\mathrm{d} G}{\mathrm{~d} p}\right|_{p=p_{c}, k=1} & =\left.\left(k^{\gamma}+\gamma p k^{\gamma-1} \frac{\partial k}{\partial p}\right)\right|_{p=p_{c}, k=1} \\
& =1+\gamma p_{c} \frac{\partial k}{\partial p}\left(p_{c}\right) .
\end{aligned}
$$

470 if

$$
\left|1+\gamma p_{c} \frac{\partial k}{\partial p}\left(p_{c}\right)\right|<1 .
$$

With the constraint of Eq. (13), this inequality reduces to

$$
\left|\gamma p_{c} \frac{\partial k}{\partial p}\left(p_{c}\right)\right|<2
$$

This suggests that it is possible to stabilise an unstable search by reducing the absolute value of the exponent $\gamma$. This is illustrated by Fig. 5, which shows the effect of the choice of the exponent $\gamma$ on the bifurcation diagram for the subcritical $\alpha$ eigenvalue search. Clearly, reducing the absolute value of $\gamma$ helps in stabilising the search: the search with $\gamma=-1$ fails to converge for $1 / k_{\infty}>3$, while the search with $\gamma=-1 / 2$ converges up to $1 / k_{\infty}=5$. However, bifurcations still appear for larger values of $1 / k_{\infty}$. More generally, for arbitrary $\gamma$ it can be shown that the map first bifurcates at

$$
k_{\infty}=\frac{\gamma}{2+\gamma}
$$

By taking sufficiently small $\gamma$, the stability of the eigenvalue search can be extended to deep sub-critical systems. However, this will come at the price of progressively slowing down the convergence of the power iteration coupled to the update rule. This regularization technique has been successfully applied also to the case of $\alpha$ eigenvalue search for the finite-size rod model, as illustrated in Fig. 2. by setting $\gamma=-1 / 2$ it has been possible to extend the range of convergence of the algorithm well below the value of $k$ which led to the failure detected for $\gamma=-1$.

The option of setting the value of the constant $\gamma$ has been implemented in TRIPOLI- $4{ }^{\circledR}$ as an effective means of stabilizing the albedo eigenvalue search for sub-critical systems. In order to illustrate the effectiveness of the parameter $\gamma$, we have re-run the sub-critical assembly configuration considered in the previous section by setting $\gamma=-1 / 2$ : the simulation results are displayed in Fig. 4 as 
a function of the power iteration generations. In this case, the convergence is ensured, and the oscillations are suppressed.

Another possibility to stabilize the eigenvalue search consists in resorting to operator-shift techniques, as suggested for instance in (Cullen et al., 2003; Yamamoto and Miyoshi, 2003; Ye et al., 2006). Let us illustrate this point in the case of $\alpha$ eigenvalues: instead of solving

$$
\mathcal{L} \varphi_{k(\alpha)}+\frac{\alpha}{v} \varphi_{k(\alpha)}=\frac{1}{k(\alpha)} \mathcal{F} \varphi_{k(\alpha)}
$$

we can solve the shifted equation

$$
\mathcal{L} \varphi_{k(\alpha)}=\frac{1}{k(\alpha)} \mathcal{F} \varphi_{k(\alpha)}-\frac{1}{k(\alpha)} \frac{\alpha}{v} \varphi_{k(\alpha)},
$$

for $k(\alpha)=1$, where the term containing the $\alpha$ eigenvalue is formally treated as a production operator. For infinite systems, e.g., this would yield the modified relation

$$
k(\alpha)=k_{\infty}-\alpha / \alpha_{*},
$$

which is to be compared with the original Eq. (22). Correspondingly, the update rule for the sub-critical case would become

$$
\alpha^{(m+1)}=\frac{\alpha^{(m)}}{k_{\infty}-\alpha^{(m)} / \alpha_{*}}
$$

which is easily seen to be stable for any $k_{\infty}$ and $\alpha_{*}$.

It has been observed that the shift given in Eq. 61) is not convenient for the Monte Carlo implementation of the power iteration, and might lead to other numerical instabilities (Yamamoto and Miyoshi, 2003; Ye et al., 2006). Operatorshift strategies ensuring the convergence of the eigenvalue search and better suited to Monte Carlo methods have been successfully tested in (Yamamoto and Miyoshi, 2003; Ye et al., 2006, Zoia et al., 2014, 2015; Nauchi, 2014).

A closely related approach consists in applying eigenvalue-shift techniques, which can be again seen as a clever way to recast the search problem into a numerically stable form. For instance, in the case of $\alpha$ eigenvalues Eq. (60) can be rewritten as

$$
\mathcal{L} \varphi_{k(\eta)}+\frac{\eta}{v} \varphi_{k(\eta)}+\frac{\tilde{\alpha}}{v} \varphi_{k(\eta)}=\frac{1}{k(\eta)} \mathcal{F} \varphi_{k(\eta)},
$$

where the search parameter is now $\eta=\alpha-\tilde{\alpha}$. Here the eigenvalue shift $\tilde{\alpha}$ must be chosen under the constraint that $\tilde{\alpha}$ should be smaller than the dominant 
$\alpha$ eigenvalue, so that $\eta>0$. Under these provisions, the update rule becomes $\eta^{(m+1)}=k\left(\eta^{(m)}\right) \eta^{(m)}$, which is stable.

It remains to discuss whether other forms of the update rule are intrinsically resilient to failure by bifurcation. For instance, update rules of the kind

$$
\alpha^{(m+1)}=\alpha^{(m)}+C \cdot\left(k\left(\alpha^{(m)}\right)-1\right)
$$

where $C$ is a constant, were proposed in the literature for $\alpha$ eigenvalue searches (Hill, 1983). It is easily seen that this equation satisfies the requirements (10) and (11)

525 for a suitably chosen sign of $C$. However, Eq. (65) is not immune from the emergence of bifurcations in deep sub-critical systems, as shown by analysing the stability of its fixed point as above. The failure of the eigenvalue search performed with Eq. (65) was indeed numerically noticed by Hill (1983), but the phenomenon was not clearly elucidated at the time.

Within the class of simple update rules $G(p, k)$ considered in this paper, it seems hardly feasible to unconditionally ensure the stability of the search algorithms for any $k(p)$. To this end, more complex schemes should be introduced, such as two-point update laws (Cho et al., 2009; Yun and Cho, 2009). However, for Monte Carlo applications these approaches are very demanding in terms of CPU time and their robustness has not been rigorously proven. Stochastic approximation and sample-path methods are quite promising (Pasupathy and Kim, 2011). Investigation of these issues is left for future research.

\section{Conclusions}

In this paper we have examined the behaviour of the eigenvalue searches that emerge in several applications in reactor physics. Such problems are usually solved by resorting to a modified power iteration where the sought eigenvalue plays the role of an adjustable free parameter. The solution is found by applying the power iteration with an update rule for the parameter depending on the dominant multiplication factor of the underlying power iteration. The functional form of the update rule is chosen based on physical considerations.

We have shown that the eigenvalue search might fail for sufficiently deep subcritical systems: the update rule undergoes period-doubling bifurcations leading to a multiplicity of parameter values, with the search algorithm indefinitely oscillating between them. In order to investigate the origins of this behaviour, we have examined a class of eigenvalue problems related to a single-speed rod model, a neutron transport system where particles are only allowed to move along two directions, namely, forward and backward. The rod model is simple enough to 
obtain in some cases exact formulas, and yet retains the key physical ingredients that have motivated our investigation. Mathematical analysis of the search

\section{Acknowledgements}

TRIPOLI- ${ }^{\circledR}$ is a registered trademark of CEA. The authors wish to thank Électricité de France (EDF) for partial financial support, and express their gratitude to Dr. F. Malvagi of CEA/Saclay for fruitful discussions.

\section{References}

Azmy, Y., Sartori, E., 2010. Nuclear Reactor Kinetics: 1934-1999, Nuclear Computational Science, A Century in Review, Springer.

Brun, E., et al., 2015. Ann. Nucl. Energy 82, 151-160.

Cho, N. Z., Yun, S., Lee, J., 2009. Trans. Am. Nucl. Soc. 101, 707-710. 
Collet, P., Eckmann, J.-P., 1980. Iterated Maps on the Interval as Dynamical Systems, Birkhauser. 590 Cullen, D.E., et al., 2003. UCRL-TR-201506.

Duderstadt, J.J., Martin, W.R., 1979. Transport theory, J. Wiley and sons, New York.

Feigenbaum, M. J., 1978. J. Stat. Phys. 19, 25-52.

Feigenbaum, M. J., 1979. J. Stat. Phys. 21, 669-706.

Feigenbaum, M. J., 1983. Physica D: Nonlinear Phenomena 7, 16-39.

595 Gelbard, E.M., Lell, R., 1976. Nucl. Sci. Eng. 63, 9-23.

Goad, W., Johnston, R., 1959. Nuc. Sci. Eng. 5, 371-375.

Hill, T.R., 1983. LA-9602-MS (UC-32).

Householder, A. S., 1970. The numerical treatment of a single nonlinear equation, McGraw-Hill, New York.

Larsen, E.W., Zweifel, P.F., 1974. J. Math. Phys. 15, 1987-1997.

Lewis, E. E., Miller, W. F. Jr., 1984. Computational Methods of Neutron Transport, J. Wiley and Sons.

Lux, I., Koblinger, L., 1991. Monte Carlo particle transport methods: Neutron and photon calculations, CRC Press, Boca Raton.

605 May, R. M., 1976. Nature 261, 459-467.

Montagnini, B., Pierpaoli, V., 1971. Transp. Theory and Stat. Phys. 1, 59-75.

Nauchi, Y., 2014. In Proceedings of the SNA+MC 2013 conference, Paris, France.

Nolen, S.D., Adams, T.R., Sweezy, J.E., 2012. LA-UR-12-25458.

Ortega, J. M., Rheinboldt, W. C., 1970. Iterative Solution of Nonlinear Equations in Several Variables, Academic Press, New York, NY.

Pasupathy, R., S. Kim , 2011. ACM Trans. Model. Comp. Sim. 21, 19.

Rouchon, A., Zoia, A., Sanchez, R., 2017. Ann. Nucl. Energy 102, 465-475.

Traub, J. F., 1964. Iterative Methods for the Solution of Equations, Prentice-Hall, Englewood Cliffs, NJ.

615 Weinberg, A.M., 1952. Am. J. Phys. 20, 401-412.

Wing, G.M., 1962. An introduction to transport theory, Wiley, New York.

Yamamoto, T., Miyoshi, Y., 2003. In Proceedings of the 7th Int. Conf. Nuc. Crit. Safety, ICNC 2003, Tokai-mura, Japan.

Yamamoto, T., 2012a. Ann. Nucl. Energy 47, 14-20.

620 Yamamoto, T., 2012b. Ann. Nucl. Energy 50, 141-149.

Ye, T., Chen, C., Sun, W., Zhang, B., Tian, D., 2006. In Proceedings of the 2006 Symposium on nuclear data, Tokai-mura, Japan.

Yun, S., Cho, N. Z., 2010. Nucl. Eng. Technol. 42, 271-278.

Zoia, A., Brun, E., Malvagi, F., 2014. Ann. Nucl. Energy 63, 276.

625 Zoia, A., Brun, E., Damian, F., Malvagi, F., 2015. Ann. Nucl. Energy 75, 627. 